\title{
Análise da produção científica sobre recursos humanos de enfermagem no Brasil ${ }^{*}$
}

\author{
Analysis of the scientific production on nursing human resources in Brazil \\ Análisis de la producción científica sobre recursos bumanos de enfermería en Brasil
}

Leide Irislayne Macena da Costa e Silva ${ }^{1}$, Marina Peduzzi ${ }^{2}$

\section{RESUMO}

Objetivos: Identificar a produção científica nacional sobre recursos humanos de enfermagem (RHE), analisá-la e estabelecer categorias para sua classificação. Métodos: Estudo bibliográfico, de 1986 a 2003, em ampla variedade de bases de dados. Resultados: Do total de 539 produções obteve-se, após refinamento, um conjunto de 162 publicações com resumos disponíveis, completos e pertinentes ao tema. A análise mostrou a predominância da abordagem de RHE da perspectiva da administração e gerenciamento de enfermagem, com 60 (37\%) publicações; do trabalho e das práticas de enfermagem, 45 (28\%) publicações, e da educação e formação profissional, 39 (24\%). Conclusões: A pesquisa permitiu validar a tipologia das vertentes de investigação sobre RHE adotada, a atualização do estado da arte sobre o tema, no país, e a elaboração de planilha eletrônica com a produção científica identificada a ser disponibilizada por meio eletrônico para consulta pública.

Descritores: Recursos humanos de enfermagem; Enfermagem/recursos humanos; Recursos humanos em saúde; Enfermagem/organização \& administração.

\begin{abstract}
Objectives: To identify the national scientific production in human resources in nursing, to analyze this scientific production, and to establish categories for its classification. Method: A literature review was conducted in several databases. The search was limited to manuscripts published between 1986 and 2003. Results: Initially a total of 539 publications were retrieved. Screening of the abstract of these publications generated a final sample of 162 publications addressing human resources in nursing. The analysis of these publications showed that $60(37 \%)$ addressed human resources in nursing from administrative and managerial perspective, 45 (28\%) from nursing practice perspective, and 39 (24\%) from educational and professional training perspective. Conclusion: The study allowed the validation of typology adopted for the investigation of human resources in nursing, the updating of the state of the science in human resources in nursing in the country, and the elaboration of an electronic table containing the scientific production about the human resource in nursing, which will be made available for public consultation.
\end{abstract}

Keywords: Nursing staff; Health manpower; Nursing/ manpower; Nursing/organization \& administration.

\section{RESUMEN}

Objetivos: Identificar la producción científica nacional sobre recursos humanos de enfermería (RHE), analizarla y establecer categorías para su clasificación. Métodos: Estudio bibliográfico, realizado entre 1986 y 2003, en una amplia gama de bases de datos. Resultados: Del total de 539 producciones se obtuvo, después de un refinamiento, un conjunto de 162 publicaciones con resúmenes disponibles, completos y pertinentes al tema. El análisis mostró el predominio del abordaje del RHE bajo la perspectiva de la administración y gerencia de enfermería, con $60(37 \%)$ publicaciones; del trabajo y de las prácticas de enfermería, 45 (28\%) publicaciones, y de la educación y formación profesional, 39 (24\%). Conclusiones: La investigación permitió validar la tipología de las vertientes de investigación sobre el RHE adoptado, la actualización del estado del arte sobre el tema, en el país, y la elaboración de una planilla electrónica con la producción científica identificada a ser puesta a disposición, en el medio electrónico, para consulta pública.

Descriptores: Personal de enfermería; enfermería/recursos humanos; recursos humanos en salud; Enfermería/organización \& administración.

\footnotetext{
* O estudo foi desenvolvido com apoio do Programa de Iniciação Científica (PIBIC/CNPq/USP-2003-2004) e apoio do Ministério da Saúde como sub-projeto da pesquisa "Avaliação do impacto do Profae na qualidade dos serviços de saúde".

${ }^{1}$ Enfermeira graduada pela Escola de Enfermagem da Universidade de São Paulo (SP), Brasil.

${ }^{2}$ Professora Doutora da Escola de Enfermagem da Universidade de São Paulo, Departamento de Orientação Profissional (SP), Brasil.
} 


\section{INTRODUÇÃO}

Observa-se um consenso entre gestores e trabalhadores de saúde, que a formação, o desempenho e a gestão de recursos humanos afetam profundamente a qualidade dos serviços prestados e o grau de satisfação dos usuários, bem como o reconhecimento de que os recursos humanos em saúde constituem elemento crítico para alcançar a finalidade e os objetivos dos serviços de saúde $^{(1)}$. Com base nestas apreciações e no contingente de profissionais de enfermagem que representam $60,2 \%$ dos vínculos de emprego de profissionais de saúde ${ }^{(2)}$, considera-se necessário conhecer a produção teórica sobre a temática recursos humanos de enfermagem (RHE). Assim, neste artigo, apresentam-se os resultados de uma pesquisa bibliográfica cujos objetivos foram: identificar a produção científica nacional sobre esse tema, no período de 1986 a 2003, analisar e classificar as publicações selecionadas.

$\mathrm{Na}$ América Latina e no Brasil, os estudos sobre os recursos humanos em saúde contam com importantes contribuições desde os anos 60, permitindo estabelecer vertentes de investigação sistematizadas na literatura nacional.

Os estudos sobre educação médica e educação em enfermagem inauguram a tradição de pesquisa sobre recursos humanos em saúde e enfermagem, configurando a primeira vertente de pesquisa na área, denominada formação profissional. Outras duas importantes abordagens também aplicáveis à área de enfermagem são organização social das práticas de saúde ou trabalho em saúde e força de trabalho em saúde ${ }^{(3-4)}$. Segundo Nogueira ${ }^{(1)}$ : "a abordagem do trabalho em saúde ganhou evidência no Brasil em duas variantes importantes: uma, de conformação mais sociológica, iniciada pelas investigações de (1975) sobre o mercado de trabalho do médico em São Paulo, com dados primários; e outra, mais econômico-demográfica, feita a partir de dados dos censos e de inquéritos estatísticos nacionais da qual são exemplos os estudos organizados por Médici (1987) e Machado (1992) (Nogueira, 2002: 257).

Embora exista essa sistematização dos estudos sobre recursos humanos em saúde e abundante produção, desde os anos 80 vem sendo apontada a necessidade de intensificar os investimentos em pesquisa nesta área, pois identifica-se que grande parte da produção teórica não está em conformidade com os padrões de cientificidade que são sancionados pelas agências de fomento à pesquisa $^{(3)}$. Como apontado acima, a organização social das práticas e do trabalho em saúde representa importante linha de pesquisa sobre os recursos humanos. No Brasil, os estudos do trabalho de enfermagem foram iniciados em meados dos anos 80 e constituem uma expressiva contribuição para a compreensão da enfermagem como prática especializada e simultaneamente articulada às demais práticas de saúde ${ }^{(5)}$.
No trabalho de enfermagem, e particularmente do enfermeiro, cabe destacar a dimensão gerencial, seja do gerenciamento do cuidado ou da gerência de unidade e serviço. Assim sendo, a área de enfermagem desenvolveu um conjunto de estudos e de intervenções práticas denominado administração em enfermagem que contempla as questões referentes aos recursos humanos de enfermagem, considerada neste estudo, como vertente de investigação de RHE.

Com base no referencial teórico adotado, foram selecionadas três vertentes de pesquisa sobre RHE que constituem as categorias de análise do material empírico, isto é, as publicações sobre RHE produzidas no período de 1986 a 2003. Assim, cunhou-se como primeira proposta de classificação as seguintes categorias: RHE da perspectiva da educação e formação profissional, RHE da perspectiva do trabalho/prática de enfermagem e RHE da perspectiva da administração/gerenciamento de enfermagem.

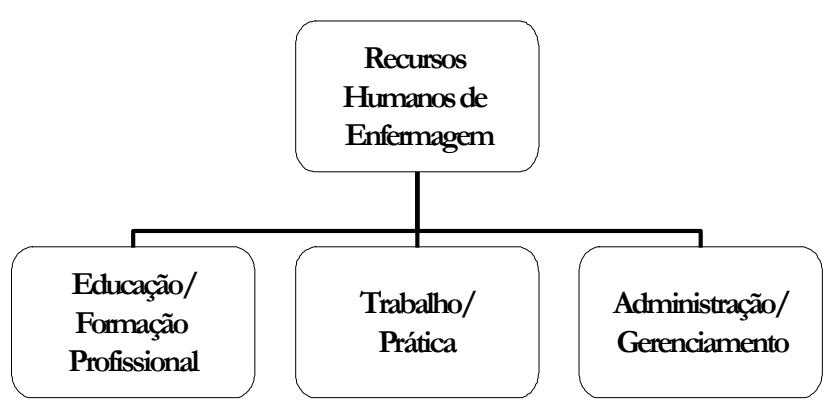

Figura 1 - Categorias de análise das publicações

\section{MÉTODOS}

A revisão sistemática consiste em um procedimento metódico de busca bibliográfica que foi definido por um grupo de cientistas reunidos em 1995, em Potsdam (Alemanha), e define-se pela aplicação de estratégias que limitem os vieses da seleção de artigos, avaliem com espírito crítico os artigos e sintetize os estudos relevantes em um tópico ${ }^{(6)}$. Embora a revisão sistemática seja indicada para estudos de questões clínicas específicas, tal como na prática baseada em evidências, fornece importantes indicativos no que se refere a limitar os possíveis vieses na revisão de literatura apresentada neste artigo.

Segundo Galvão et $\mathrm{al}^{\left({ }^{()}\right)}$: "a revisão tradicional da literatura há muito tempo vem sendo criticada, devido à ausência de métodos explícitos e rigorosa padronização das técnicas empregadas. Em resposta a essa situação, o desenvolvimento de métodos seguros para serem utilizados na revisão sistemática tem ocorrido de forma considerável (Galvão et al, 2004: 554). 
Tomando como base o procedimento de revisão sistemática foi realizada exaustiva busca do material bibliográfico através de uma estratégia ampla de busca nas bases eletrônicas de dados. Em se tratando de uma pesquisa sobre recursos humanos de enfermagem no Brasil, a busca bibliográfica foi realizada em todas as bases de dados nacionais e latino-americanas disponíveis e pertinentes à temática Recursos Humanos (LILACS, Perienf, DEDALUS - global, BDENF, PAHO, AdSaude, SIDORH, MS, e TEXTOC)* e nos catálogos do CEPEn, no período de dezembro de 2003 a janeiro de 2004.

Foi selecionado o período de 1986 a 2003 para a pesquisa porque, segundo estudo anterior ${ }^{(7)}$, concentra as publicações com o descritor RHE.

Para a seleção e posterior exclusão das publicações repetidas, das internacionais e daquelas sem resumo disponível ou com resumo incompleto, foi elaborada uma planilha eletrônica** da produção científica constando todas as publicações identificadas, segundo as seguintes variáveis: autor, título, fonte, ano, base de dados, publicações sem resumo disponível, com resumo incompleto e com resumo não pertinente à temática de estudo.

A identificação das publicações repetidas seguiu a ordem das bases de dados LILACS, PeriEnf, DEDALUS (global), BDENF, PAHO, AdSaude, SIDORH, MS, TEXTOC e CEPEn e foram consideradas repetidas quando possuíam, primeiramente o mesmo autor e, secundariamente, o mesmo título, mesmo sendo publicadas em fontes e anos diferentes. Quando as publicações eram repetidas na mesma base, excluíam-se as mais recentes. Quando as publicações eram repetidas entre as bases, utilizava-se a ordem crescente das bases já descrita acima. Todas as publicações repetidas na base e entre as bases também foram excluídas, usando o critério da ordem crescente das bases.

Identificaram-se publicações com o mesmo autor, cujos títulos apresentavam uma discreta modificação. Também foram observadas publicações nas quais havia autor e/ou autores diferentes, porém com o mesmo título. Todas estas publicações foram consideradas diferentes.

A análise das publicações iniciou com a leitura dos

\footnotetext{
* Bases bibliográficas consultadas: LILACS (Literatura Latino Americana e do Caribe em Ciências da Saúde); Perienf (Base de Dados de Literatura Periódica em Enfermagem); DEDALUS (global) - Base de Dados Bibliográficas da USP; BDENF (Base de Dados em Enfermagem); PAHO (Acervo da Biblioteca da Organização Pan-Americana da Saúde); AdSaude (Administração de Serviços de Saúde); SIDORH (Recursos Humanos em Saúde); MS (Acervo da Biblioteca do Ministério da Saúde) e TEXTOC (Textos Completos). CEPEn - Centro de Estudos e Pesquisa em Enfermagem da Associação Brasileira de Enfermagem.

** A planilha eletrônica foi elaborada utilizando o software Excel 2002, versão 7.0, desenvolvido pela Microsoft ${ }^{\mathbb{R}}$.
}

resumos tomando como base a primeira proposta de classificação dessa literatura, definida a partir das vertentes de investigação sobre recursos humanos em saúde e enfermagem apresentadas inicialmente.

O início da análise permitiu identificar resumos incompletos. Foram considerados incompletos os resumos que não apresentavam de forma clara e objetiva o tema, os objetivos e os resultados da respectiva publicação. Esta decisão foi tomada visto que a leitura do material mostrou que a maior parte dos resumos não incluía a totalidade dos aspectos a serem contemplados, is to é: tema, objetivo, metodologia, resultados e conclusões.

Dentre os resumos completos foram identificadas publicações não pertinentes à temática de recursos humanos de enfermagem, o que levou à exclusão dessa produção.

Considerado o conjunto de publicações com resumo completo e pertinente ao tema, procedeu-se à análise e classificação com base nas categorias propostas inicialmente. Porém, a análise do material mostrou ser necessária a utilização de novas categorias, bem como de sub-categorias para aquelas já previstas, expressando de forma mais fidedigna os temas e a abordagem trabalhada na produção teórica. As novas categorias e sub-categorias são apresentadas, a seguir, nos resultados e discussão. Os resumos classificados como "outros" são aqueles para os quais não foi possível um consenso entre as pesquisadoras na classificação ou que não puderam ser classificados, pois o resumo não permitia uma compreensão clara e objetiva.

\section{RESULTADOS E DISCUSSÃO}

A classificação da produção científica permite observar que as vertentes de pesquisa sobre recursos humanos em saúde também estão presentes na área de enfermagem, demonstrando a consolidação de linhas como: educação/ formação profissional e trabalho/prática de enfermagem. Também se pode observar que vêm sendo produzidos e divulgados estudos sobre temas referentes à administração e gerenciamento de enfermagem, tal como assinalados por Schraiber e Peduzzi ${ }^{(8)}$ acerca das investigações no campo de RHS.

A consulta a todas as bases de dados, no período de 1986 a 2003, identificou um total de 539 publicações com repetições e 395 sem repetições. Dentre as últimas, havia 240 com resumos disponíveis. A leitura desses resumos permitiu observar que das 240 publicações, apenas 209 (87\%) possuíam resumos completos e 31 (13\%) incompletos. Do total de resumos completos, apenas 162 $(77,5 \%)$ eram pertinentes à temática RHE, ou seja, se referiam a estudos sobre recursos humanos de enfermagem e $47(22,5 \%)$ não eram pertinentes, embora 
constassem nas bases de dados com o descritor pesquisado. Portanto, 162 resumos constituíram o total para análise e classificação.

Quanto ao tipo de publicação, do total de 162 resumos analisados, 95 (59\%) são artigos de periódicos; 63 (39\%) são pesquisas desenvolvidas em curso de pós-graduação, sendo 37 dissertações de mestrado e 26 teses de doutorado; $3(1,9 \%)$ são teses de livre docência e uma $(0,6 \%)$ publicação refere-se a um projeto da Organização Pan-Americana da Saúde.

Dentre as 162 publicações, 60 (37\%) foram classificadas na categoria RHE da perspectiva da administração e gerenciamento de enfermagem, 39 (24\%) foram classificadas como RHE da perspectiva da educação e formação profissional, 45 (28\%) foram classificadas como RHE da perspectiva do trabalho e prática de enfermagem, $10(6 \%)$ como RHE da perspectiva do perfil profissional, 4 (2,5\%) como RHE da perspectiva da motivação e/ou satisfação no trabalho e $4(2,5 \%)$ na categoria outros (Tabela 1$)$. Portanto, observa-se a predominância de publicações sobre RHE na abordagem admi-nistrativa, seguida do enfoque do trabalho e, em terceiro lugar, do enfoque da educação.

Tabela 1 - Distribuição das publicações sobre RHE no período de 1986 a 2003, segundo categorias de análise. Brasil, 2003 e 2004.

\begin{tabular}{lcc}
\hline $\begin{array}{l}\text { Categorias de análise das publicações sobre } \\
\text { RHE }\end{array}$ & No & $\mathbf{\%}$ \\
\hline $\begin{array}{l}\text { RHE da perspectiva da administração/ } \\
\text { gerenciamento de enfermagem }\end{array}$ & 60 & 37,0 \\
$\begin{array}{l}\text { RHE da perspectiva do trabalho/prática de } \\
\text { enfermagem }\end{array}$ & 45 & 28,0 \\
$\begin{array}{l}\text { RHE da perspectiva da educação e formação } \\
\text { profissional }\end{array}$ & 39 & 24,0 \\
$\begin{array}{l}\text { RHE da perspectiva do perfil profissional } \\
\text { RHE da perspectiva da motivação e/ou }\end{array}$ & 10 & 6,0 \\
$\begin{array}{l}\text { satisfação no trabalho } \\
\text { Outros }\end{array}$ & 4 & 2,5 \\
\hline Total & 4 & 2,5 \\
\hline
\end{tabular}

Para as três categorias de análise que concentram a maior parte das publicações, foi necessário definir subcategorias para uma classificação mais fidedigna e precisa da produção teórica. Assim sendo, a categoria RHE da perspectiva da administração foi refinada com subcategorias baseadas nos instrumentos do processo de trabalho administrar/gerenciar em enfermagem, quais sejam: dimen-sionamento de pessoal, recrutamento e seleção, educação continuada, avaliação de desempenho e outras que expressam os diferentes meios utilizados no processo de trabalho gerencial do enfermeiro.

Observa-se a relevância de publicações sobre administração e gerenciamento de enfermagem. Estudo realizado por Ermel, Fracolli(i) ${ }^{(9)}$ ue teve como objetivo identificar como a gerência tem sido abordada na produção científica nacional, no período de 1989 a 1999, refere que no campo científico da enfermagem, temas relacionados às questões gerenciais são freqüentemente encontrados, uma vez que as funções administrativas estão fortemente relacionadas ao trabalho do enfermeiro. Segundo as autoras, a leitura desses trabalhos revela que, de modo geral, o trabalho da gerência desenvolvido pela enfermeira é fortemente influenciado pelas concepções de organização do trabalho baseadas nos princípios de Taylor, Fayol e nas Teorias Administrativas Clássicas, contudo, propostas gerenciais baseadas nas concepções de organização tecnológica do trabalho; gerência participativa e comunicativa; na teoria das organizações e no pensamento estratégico vêm, cada vez mais, conquistando espaços nos trabalhos desenvolvidos dentro do campo da enfermagem.

No presente estudo observa-se a relevância das subcategorias dimensionamento do pessoal de enfermagem, educação continuada em enfermagem e avaliação de desempenho em enfermagem, visto concentrarem o maior número de publicações nesta categoria. Em contrapartida, verifica-se a escassez de publicações sobre temáticas como supervisão em enfermagem, parceria docente-assistencial e avaliação dos serviços de saúde, que também são relevantes para o exercício da gerência local ou da gestão de serviços (Tabela 2).

Entende-se que o tema dimensionamento de pessoal é relevante para a área de enfermagem, uma vez que, freqüentemente, observam-se problemas no quadro de pessoal de enfermagem das instituições de saúde, inclusive a escassez de recursos humanos, tanto na rede pública como na rede privada de serviços. Embora, a força de trabalho de enfermagem represente $60,2 \%$ do total de vínculos de empregos de profissionais de saúde ${ }^{(2)}$, ainda assim constata-se um quadro insuficiente de pessoal em algumas instituições, à medida que esta área de trabalho responde por ações executadas continuamente, sobretudo nos serviços hospitalares.

Além disso, a necessidade de desenvolvimento de pessoal tem sido reforçada pelos avanços tecnológicos e pelas mudanças no mundo do trabalho, que levam as organizações e os indivíduos a adquirir e atualizar conhecimentos. Nesse sentido, destacam-se competências como comunicação e interação social, que constituem ferramentas intrínsecas do trabalho na área de enfermagem, que são adquiridas ao longo da vida e de processos educativos e não em capacitações pontuais, o que remete à necessidade de investimentos no que se poderia, de forma mais geral, denominar "educação permanente em serviços"(10-11).

Os resultados encontrados mostram a necessidade de investigar o próprio processo de trabalho gerencial de enfermagem e não apenas os instrumento desse trabalho, 
sobretudo as concepções teóricas e os projetos éticopolítico adotados pelas práticas de gestão e de gerência de enfermagem, bem como os conflitos existentes entre a dimensão assistencial e gerencial do processo de trabalho de enfermagem, que são aqui entendidas como indissociáveis. Também se evidencia a necessidade de pesquisar sobre o gerenciamento e a gestão em saúde, analisando as suas peculiaridades por referencial ao campo da administração e as mediações entre os conhecimentos produzidos por este campo e o setor saúde.

Tabela 2 - Distribuição das publicações sobre RHE no período de 1986 a 2003 na perspectiva da administração e gerenciamento de enfermagem. Brasil, 2003 e 2004.

\begin{tabular}{lcc}
\hline $\begin{array}{l}\text { Sub-Categorias: RHE da Perspectiva da } \\
\text { Administração e Gerenciamento de } \\
\text { Enfermagem }\end{array}$ & No & $\mathbf{\%}$ \\
\hline $\begin{array}{l}\text { Dimensionamento do pessoal de enfermagem } \\
\text { Avaliação de desempenho de pessoal de } \\
\text { enfermagem }\end{array}$ & 16 & 27,0 \\
Educação continuada em enfermagem & 12 & 20,0 \\
Organização dos serviços de saúde/RHE & 9 & 15,0 \\
Rotatividade de pessoal de enfermagem & 6 & 10,0 \\
Liderança em enfermagem & 5 & 8,0 \\
Recrutamento e seleção de pessoal de & 3 & 5,0 \\
enfermagem & & \\
Absenteísmo em enfermagem & 3 & 5,0 \\
Supervisão em enfermagem & 2 & 3,0 \\
Parceria docente-assistencial & 1 & 1,7 \\
Avaliação dos serviços de saúde/RHE & 1 & 1,7 \\
Escala de pessoal de enfermagem & 1 & 1,7 \\
\hline Total & 1 & 1,7 \\
\hline
\end{tabular}

Quanto à categoria RHE da perspectiva do trabalho e prática de enfermagem, que aparece em segundo lugar, foram identificadas $45(28 \%)$ publicações, predominando as temáticas "saúde do trabalhador" e "mercado de trabalho" (Tabela 3).

Observa-se, assim, a existência de uma tradição consolidada de estudos sobre saúde do trabalhador na área de enfermagem, provavelmente associada ao grande contingente da força de trabalho de enfermagem no país e às características do seu trabalho que leva a processos de desgastes, comprometendo a saúde dos trabalhadores e a produtividade dos serviços.

Ainda associada à temática saúde do trabalhador, verificam-se $4(9 \%)$ publicações sobre a sub-categoria "psicodinâmica do trabalho" que, com base em uma abordagem teórica específica, também investiga aspectos relacionados ao sofrimento provocado pelo trabalho de enfermagem (Tabela 3).

A concepção da enfermagem como trabalho pressupõe a apreensão articulada dos elementos que o constituem e caracterizam como trabalho especializado, ou seja: o objeto de trabalho, os instrumentos e a finalidade, articulada a análise do processo de divisão técnica e social do trabalho, que foi intenso no Brasil. Nesse sentido, destacam-se as $6(13 \%)$ e $4(9 \%)$ publicações, respectivamente nas sub-categorias "trabalho/prática do enfermeiro" e "trabalho/prática do pessoal de enfermagem" (Tabela 3).

Como já assinalado, as vertentes de investigação sobre RHS e RHE contemplam tanto a organização das práticas ou o trabalho, quanto os aspectos relacionados à força de trabalho, concebida como a capacidade de trabalho que os trabalhadores disponibilizam no mercado de trabalho, numa relação de compra e venda mediada por um preço ${ }^{(12)}$. Assim, podem-se relacionar, também nesta perspectiva teórica, as publicações sobre mercado de trabalho e força de trabalho, que aparecem, respectivamente, com $8(18 \%)$ e $5(11 \%)$ no total da produção científica identificada (Tabela 3 ).

Tabela 3 - Distribuição das publicações sobre RHE no período de 1986 a 2003 na perspectiva do trabalho e prática de enfermagem. Brasil, 2003 e 2004.

\begin{tabular}{lcr}
\hline $\begin{array}{l}\text { Sub-Categoria: RHE da Perspectiva do } \\
\text { Trabalho e Prática de Enfermagem }\end{array}$ & $\mathbf{N}^{\mathbf{0}}$ & $\mathbf{\%}$ \\
\hline $\left.\mathbf{c}_{\mathbf{1}}\right)$ Saúde do trabalhador de enfermagem & 12 & 27,0 \\
& & \\
$\left.\mathbf{c}_{2}\right)$ Mercado de trabalho de enfermagem & 8 & 18,0 \\
$\left.\mathbf{c}_{3}\right)$ Trabalho/prática do enfermeiro & 6 & 13,0 \\
$\left.\mathbf{c}_{4}\right)$ Política de saúde/ política de RHE & 6 & 13,0 \\
$\left.\mathbf{c}_{5}\right)$ Força de trabalho de enfermagem & 5 & 11,0 \\
$\left.\mathbf{c}_{6}\right)$ Trabalho/prática do pessoal de enfermagem & 4 & 9,0 \\
$\left.\mathbf{c}_{\mathbf{7}}\right)$ Psicodinâmica do trabalho & 4 & 9,0 \\
\hline Total & $\mathbf{4 5}$ & $\mathbf{1 0 0 , 0}$ \\
\hline
\end{tabular}

Em relação à categoria RHE da perspectiva da educação e formação profissional, na qual aparecem 39 $(24 \%)$ publicações, observa-se uma distribuição mais eqüitativa das sub-categorias, ou seja: $10(26 \%)$ referemse à capacitação em temáticas específicas, $10(26 \%)$ à educação superior, 11 (28\%) educação profissional e 6 $(15 \%)$ à política de formação de recursos humanos de enfermagem. Contudo, chama atenção a reduzida produção sobre tecnologias aplicadas ao ensino e análise de currículo (Tabela 4).

Desde o início do processo de reflexão sistematizada e das pesquisas sobre as questões de RHS e RHE, nos anos 60 , a abordagem da educação e formação profissional predomina. Mesmo com a introdução das demais vertentes de pesquisa, trabalho em saúde, força de trabalho e administração de enfermagem, o enfoque na educação permanece. 
O contexto particular em que emerge a questão da educação e formação profissional de enfermagem,

Tabela 4 - Distribuição das publicações sobre RHE no período de 1986 a 2003 na perspectiva da educação e formação profissional. Brasil, 2003 e 2004.

\begin{tabular}{lcr}
\hline $\begin{array}{l}\text { Sub-Categoria: RHE da Perspectiva da } \\
\text { Educação e Formação Profissional }\end{array}$ & $\mathbf{N}^{\mathbf{o}}$ & $\mathbf{\%}$ \\
\hline Educação profissional & 11 & 28,0 \\
Educação superior & 10 & 26,0 \\
Formação de RHE em temática específica & 10 & 26,0 \\
Política de formação de RHE & 6 & 15,0 \\
Tecnologia aplicada ao ensino & 1 & 2,5 \\
Análise de currículo & 1 & 2,5 \\
\hline Total & $\mathbf{3 9}$ & $\mathbf{1 0 0 , 0}$ \\
\hline
\end{tabular}

associado à intensa dinâmica de divisão do trabalho de enfermagem, pode justificar a distribuição eqüitativa entre publicações que se referem à educação superior e educação profissional. Nesse sentido, lembra-se que $52,7 \%$ dos vínculos de emprego em saúde são ocupados por trabalhadores de enfermagem de nível médio ${ }^{(2)}$, de modo que a temática educação em serviço aparece tanto nas publicações de RHE da perspetiva da administração, na sub-categoria educação continuada (Tabela 2) quanto na produção teórica sobre RHE da perspectiva da educação e formação profissional (Tabela 4).

No que se refere à educação em serviço, destacam-se as publicações identificadas na sub-categoria "Formação de RHE em temáticas específicas", dentre as quais predominam os temas DST/AIDS e aleitamento materno.

Finalmente, cabe comentar sobre as publicações identificadas na busca, mas que não puderam ser classificadas nas três categorias previstas inicialmente. Dez $(6 \%)$ publicações foram classificadas como RHE da perspectiva do perfil profissional, pois apresentavam uma caracterização de grupos de trabalhadores de enfermagem em serviços específicos. Também foram encontradas $4(2,5 \%)$ publicações categorizadas como RHE da perspectiva da motivação e/ou satisfação no trabalho, visto não se configurarem como estudos sobre a psicodinâmica do trabalho, embora tratassem da satisfação ou motivação para o exercício profissional. E, por fim, $3(1,8 \%)$ publicações foram classificadas na categoria "outros" (Tabela 1).

\section{CONCLUSÃO}

A revisão da literatura sobre recursos humanos de enfermagem abarcou um período de 17 anos, de 1986 a 2003, e mostrou a predominância da abordagem do tema da ótica da administração de enfermagem, seguida do estudo do trabalho e das práticas de enfermagem e, por fim, da educação e formação profissional, esta contempla tanto o ensino superior, quanto à educação profissional de nível médio e a educação de trabalhadores em serviços de saúde.

Desta forma, a pesquisa bibliográfica empreendida, permitiu validar a tipologia das temáticas de investigação sobre RHE adotada. Destaca-se que esta tipologia foi elaborada com base nas vertentes já sistematizadas sobre RHS e no reconhecimento da configuração de uma área específica de atuação e pesquisa de enfermagem, representada pela administração em enfermagem.

A presente pesquisa foi conduzida com rigor metodológico, buscando minimizar os possíveis vieses presentes nos estudos dessa natureza, e pautou-se em referencial teórico consagrado no campo de recursos humanos em saúde, atualizando o "estado da arte" sobre recursos humanos de enfermagem, no país, nas últimas duas décadas. Também resultou na elaboração de uma planilha eletrônica da produção científica identificada, que configura um instrumento coletivo de consulta disponível por meio eletrônico para os pesquisadores da área.

\section{REFERÊNCIAS}

1. Nogueira RP. Resultado do estudo de avaliação de tendências e prioridades sobre recursos humanos em saúde. In: Brasil. Ministério da Saúde. Política de recursos humanos em saúde [sítio na Internet] Brasília: Ministério da Saúde; 2002. p. 31-44. disponível em: http://www.bra.ops-oms.org/sistema/arquivos/ politrh.pdf

2. Girardi SN, Carvalho CL. Mercado de trabalho e regulação das profissões de saúde. In: Negri B, Faria R, Viana ALD, organizadores. Recursos humanos em saúde: política, desenvolvimento e mercado de trabalho. Campinas: Unicamp. IE.; 2002. p. 221-51.

3. Brasil. Ministério da Saúde. A investigação sobre Recursos Humanos em Saúde:relatório de seminário. Brasília: Ministério da Saúde; Coordenação Geral de Desenvolvimento de Recursos Humanos para o SUS; 1993.

4. Peduzzi M, Schraiber LB. Workshop: mapeamento de projetos de pesquisa e de intervenções sobre recursos humanos em saúde, no âmbito nacional. Interface Comum Saúde Educ. 2000; 4(7):149-52.

5. Almeida MCP, Mishima SM, Peduzzi M. A pesquisa em enfermagem fundamentada no processo de trabalho: em busca da compreensão e qualificação da prática de enfermagem. In: $51^{\circ}$ Congresso Brasileiro de Enfermagem; 1999; Florianópolis. Anais. Florianópolis: Associação Brasileira de Enfermagem - Seção SC; 2000. p. 259-77.

6. Galvão CM, Sawada NO, Trevizan MA. Revisão sistemática: recurso que proporciona a incorporação das evidências na pática da enfermagem. Rev Lat . 
Am. Enferm. 2004; 12(3):549-56.

7. Silva LIMC. Os recursos humanos de enfermagem da perspectiva da força de trabalho: análise da produção científica e da composição e distribuição dos trabalhadores de enfermagem [relatório final de pesquisa]. São Paulo: Escola de Enfermagem da USP; 2003.

8. Schraiber LB, Peduzzi M. Tendências e possibilidades da investigação de recursos humanos em saúde no Brasil. Educ Méd Salud. 1993; 27(3): 295-313.

9. Ermel RC, Fracolli LA. Processo de trabalho de gerência: uma revisão da literatura. Rev Esc Enferm. USP. 2003; 37(2):89-96.

10. Davini MC, Nervi L, Roschke MA. Capacitación del personal de los servicios de salud. Proyetos relacionados com los procesos de reforma sectorial.[on line] Washington-DC: Organización Panamericana da la Salud. Programa de Desarrollo de Recursos Humanos. División de Desarrollo de Sistemas y Servicios de Salud. Serie Observatorio de Recursos Humanos de Salud no 3 2002. [citado 2005 Fev 4]. Disponível em: http://www.paho.org/ Spanish/HSP/HSR/HSR02/libro3-obsrrhh.htm.

11. Brasil. Ministério da Saúde. Ato Portaria n 198/CM de 13 de fevereiro de 2004. Institui a política nacional de educação permanente em saúde como estratégia do Sistema Único de Saúde para a formação e o desenvolvimento de trabalhadores para o setor e dá outras providências [texto na Internet]. Brasília; 2004. [citado 2004 Set 15]. Disponível em: < $\underline{\text { http:// }}$ www.saúde.gov.br/saude/lvs/legislação/ portarias.htm

12. Nogueira RP. A força de trabalho em saúde. In: Médici AC, Paim ER. Textos de apoio: planejamento I: recursos humanos. Rio de Janeiro: Escola Nacional de Saúde Pública; 1987. p. 13-8. 